\section{Evaluation of the plasma microRNA levels in stage 3 premature retinopathy with plus disease: preliminary study}

T Metin', E Dinç², A Görür ${ }^{3}$, S Erdoğan ${ }^{4}$, S Ertekin ${ }^{5}$, AA Sarı ${ }^{2}, \mathrm{~L}$ Tamer $^{3}$ and Y Çelik ${ }^{6}$

\begin{abstract}
Purpose In the present study, we aimed to investigate the changes in plasma microRNA (miRNA) levels in premature infants diagnosed with premature retinopathy (ROP). Patients and methods In order to investigate the relationship of miRNAs with ROP, 13 premature infants admitted to Mersin University, Medical School, Department of Ophthalmology and diagnosed with ROP stage 3 with plus disease between January 2014-January 2015 were included in the study. Control group consisted of $\mathbf{1 5}$ premature infants with no ROP. The plasma miRNA levels were evaluated using highthroughput quantitative real-time PCR. Results The results of study demonstrated that the expression level of miR-23a and miR-200b-3p was significantly upregulated in patients with ROP when compared with the control group $(P<0.05)$. The expression level of miR-27b-3p and miR-214-3p was significantly downregulated in patients $(P<0.05)$. In addition, expression of 17 miRNA (miR-410-3p, miR-17-5p, miR-451a, miR-31-5p, miR-132-3p, miR-183-5p, miR-184, miR-222-3p, miR-296-5p, miR-200a-3p, miR-328-3p,miR-96-5p, miR-199a-5p, miR-99a5p, miR-106a-5p, miR-125b-5p, miR-155-5p) had upregulated or downregulated, but not statistically significantly different when compared with the control group.

Conclusions Our results suggest that plasma miRNA levels may alter in ROP and, some miRNAs might have an effect in the physiopathology of this disease. These molecules may have an important therapeutic role in patients who are unresponsive to antivascular endothelial growth factor therapy. However, further studies must be conducted for possible effects of miRNAs in vascular
\end{abstract}

disorders of eye such as ROP. Moreover to define the relationship of these molecules with the disease more clearly, a multicenter study including more patients is necessary. Eye (2018) 32, 415-420; doi:10.1038/eye.2017.193; published online 29 September 2017

\section{Introduction}

Retinopathy of prematurity (ROP), one of the most significant preventable childhood blindness causes is a proliferative vitreoretinopathy which is characterized with retinal ischemia and neovascularization. ${ }^{1}$ It is well known that many molecules are responsible for the physiopathology of the pathological angiogenesis observed in ROP. ${ }^{2}$ Vascular endothelial growth factor (VEGF) has been shown to have a causal role in the development of ROP. In addition, many studies are being conducted to understand the molecular mechanisms related to ROP and the role of genetics and environmental risk factors. ${ }^{3}$ Although the molecular mechanisms of ROP remain unclear, many new molecules such as microRNAs (miRNAs) may have a role in the pathophysiology of ROP.

MicroRNAs are non-protein coding RNA molecules that are a class of endogen small RNA. Number of single fibered miRNAs, which are 20-25 nucleotides long is over a thousand. ${ }^{4}$ Metabolic regulation, memorial and synaptic development, angiogenesis, regulation of gene expression, tumorigenesis, metamorphosis and growth control are some of the functions of those molecules. Due to these relations, miRNA based treatment methods are being researched for the patients who particularly have neoplastic and angiogenesis related diseases. Recent studies show that miRNAs have important functions in
${ }^{1}$ Ophthalmology Clinic, Mersin State Hospital, Mersin, Turkey

${ }^{2}$ Faculty of Medicine, Department of Ophthalmology, Mersin University, Mersin, Turkey

${ }^{3}$ Faculty of Medicine, Department of Biochemistry, Mersin University, Mersin, Turkey

${ }^{4}$ Faculty of Medicine, Department of Biostatistics, Mersin University, Mersin, Turkey

${ }^{5}$ Ophthalmology Clinic, Osmaniye State Hospital, Osmaniye, Turkey

${ }^{6}$ Faculty of Medicine, Division of Newborn, Mersin University, Mersin, Turkey

Correspondence:

E Dinç, Department of Ophthalmology, Faculty of Medicine, Mersin University, Mersin 33100, Turkey Tel: +90 32424100 00; Fax: +90 3242410005. E-mail: erdem_dinc@ hotmail.com

Received: 2 April 2017 Accepted in revised form: 27 July 2017

Published online: 29 September 2017

This study was presented as an oral presentation in the 49th Turkish National Congress of Ophthalmology, in Istanbul, Turkey, at 4-8 November 2015. 
the development of ocular vascular disease. ${ }^{4,5}$

It is known that some of the miRNA subtypes are shown to be responsible for angiogenesis on ischemic retina. ${ }^{6}$

Tea et al observed that the amount of miR-16 expression decreases after oxygen therapy in rat model of ROP. ${ }^{7}$ In this model, miR-16 expression was variable with strain fluctuations in oxygen levels, and developmental stage.

With the light of this knowledge it may be assumed that these molecules may also act a role in the physiopathology of ROP. We aim to investigate and compare the plasma miRNA levels of premature infants who had laser photocoagulation treatment for ROP with those without retinopathy. To our knowledge, this is the first study in the literature on human ROP.

\section{Materials and methods}

Thirteen premature infants (10 boys, 3 girls) without systemic pathology admitted to Mersin University, Medical School, Department of Ophthalmology and diagnosed as ROP stage 3 with plus disease between January 2014 and January 2015 were included in the study. Patient group consisted of premature infants, born before 37 weeks of pregnancy, who did not take any other treatment for ROP before and planned to have laser photocoagulation treatment for ROP stage 3 with plus disease under general anesthesia for the first time. Control patients ( 5 boys, 10 girls) were selected among otherwise healthy premature infants with no ROP detected in routine screening exams and screened for the risk factors as follows: infants born before 32 weeks of pregnancy, birth weight less than $1500 \mathrm{~g}$, born before the 37th gestational week and received oxygen therapy. Peripheral blood samples of the patient and control groups were obtained and analyzed for expression of 46 miRNAs in plasma. The investigations were approved by the Medical Ethical Review Committee of the Academic Hospital of Mersin University (2014/9), and informed consent was obtained from all patients and controls according to the Declaration of Helsinki II (1975, revised 1983), and the study adhered to the ARVO statement on human subjects.

\section{Plasma preparation and RNA isolation}

Two milliliters of peripheral blood was collected in EDTA tubes just prior to laser photocoagulation in patient group and while routine blood tests were scheduled to the control group. Blood samples were placed immediately on ice and then taken within $10 \mathrm{~min}$ to the laboratory. Samples were centrifuged at $2000 \times g$ for $10 \mathrm{~min}$. Plasma samples were transferred to a clean microcentrifuge tube followed by a second centrifugation step at $2000 \times g$ for $5 \mathrm{~min}$ at to remove cell debris and fragments. The plasma samples were aliquoted and stored at $-80^{\circ} \mathrm{C}$ until RNA isolation. Total RNA including small RNA and miRNAs isolated from plasma samples by using High Pure miRNA Isolation Kit (Roche diagnostic GmbH, Mannhein, Germany) according to the manufacturer's instructions. RNA samples were stored at $-80^{\circ} \mathrm{C}$ until cDNA reaction.

\section{Reverse transcription reaction}

Isolated RNAs were reverse-transcribed into cDNA in $5 \mu \mathrm{l}$ final reaction volumes using TaqMan MicroRNA Reverse Transcription Kit in combination with the Megaplex RT Primer (Applied Biosystems, Foster City, CA, USA). The reverse transcription (RT) reaction was used 40 cycles of $16^{\circ} \mathrm{C}$ for $120 \mathrm{~s}, 42^{\circ} \mathrm{C}$ for $60 \mathrm{~s}$ and $50{ }^{\circ} \mathrm{C}$ for $1 \mathrm{~s}$, followed by a final reverse transcriptase inactivation at $85^{\circ} \mathrm{C}$ for 5 min. cDNA samples were kept at $-80^{\circ} \mathrm{C}$ until PCR analysis.

\section{Pre-amplification}

Prior to PCR, cDNA samples were pre-amplified using the TaqMan PreAmp Master Mix 2X (Applied Biosystems) as well as the MegaPlex Human PreAmp Primer Pools Set v3.0 (Applied Biosystems). All reactions were performed as specified in the protocols of the manufacturer. PreAmp Thermal Protocol was performed using 384 well Thermal Cycler as follows: $95^{\circ} \mathrm{C}$ for $10 \mathrm{~min}, 55^{\circ} \mathrm{C}$ for $2 \mathrm{~min}$ and $72^{\circ} \mathrm{C}$ for $2 \mathrm{~min}$, followed by 18 cycles with $95^{\circ} \mathrm{C}$ for $15 \mathrm{~s}, 60^{\circ} \mathrm{C}$ for $4 \mathrm{~min}$, finally hold at $4{ }^{\circ} \mathrm{C}$.

\section{Quantitative real-time PCR}

Quantitative real-time PCR reactions (qRT-PCR) were performed using the high-throughput BioMark Real-Time PCR system (Fluidigm, South San Francisco, CA, USA). Pre-amplified cDNA samples were diluted with DNA Suspension buffer (Teknova, Hollister, CA, USA) 1:5 times. $490 \mu \mathrm{l}$ of TaqMan Universal PCR Master Mix, No AmpErase UNG, (Applied Biosystems), and $49 \mu \mathrm{l}$ of 20x GE Sample Loading Reagent (Fluidigm) mixed and pipetted into a 96 well plate as 3.85 $\mu$ l. 1:10 times diluted $3.15 \mu 1$ of Pre-amplified cDNA samples pipetted into each well and mixed. Then $5 \mu \mathrm{l}$ of this mixture pipetted into sample inlets of a 96.96 Dynamic Arrays (Fluidigm) $4.0 \mu 1$ 1:1 diluted 20X TaqMan miRNA Assays pipetted into assay inlets of a 96.96 Dynamic array (Fluidigm). The BioMark IFC controller HX (Fluidigm) was used to distribute the assay mix and sample mix from the loading inlets into the 96.96 Dynamic array reaction chambers for qRT-PCR by Fluidigm's Integrated Fluidic Circuit Technology. qRT-PCR was performed by using BioMark System by using following protocol; Hold on at $95^{\circ} \mathrm{C}$ for $10 \mathrm{~min}$ and $95^{\circ} \mathrm{C}$ for $15 \mathrm{~s}, 60^{\circ} \mathrm{C}$ for $1 \mathrm{~min}$ for 30 cycles. 


\section{Normalization and relative quantification of plasma miRNA expression}

To eliminate the normalization problem for miRNA expression in plasma, depending on the absence of stable RNA, we used the global mean normalization method for normalizing plasma miRNA expression. The miRNA expression data were normalized according to the global mean normalization strategy. ${ }^{8}$ The global mean normalization of the miRNA qRT-PCR data was performed with The Gene Global Data Analysis Center (Qiagen, Germantown, USA). The relative expression of miRNAs was calculated with the comparative delta delta $\mathrm{Ct}(\Delta \Delta \mathrm{CT})$ method. Fold regulation (FR) was calculated with equation $2^{-\Delta \Delta C t}$.

\section{Statistical analysis}

The proposed study was planned as a pilot study because there has been no similar study in the literature, and we targeted at least 10 participants in each group. This study included 13 patients and 15 controls who admitted to our clinic between January 2014 and January 2015. Statistical analysis was performed with the SPSS software package, version 11.5 for Windows (SPSS, Chicago, IL, USA). ChiSquare $\left(\chi^{2}\right)$ was used to evaluate the distribution of gender among the patients and the control subjects. The age of both groups was compared with an independent Student's $t$-test. Expression data were controlled for normal distribution with the Shapiro-Wilk test. According to the test results, miRNA expressions were not normally distributed. The Mann-Whitney $U$-test was used to detect differences in the expression of plasma miRNAs between the patients and healthy individuals. Results are expressed as mean $\pm \mathrm{SD}$. A $P$-value of $<0.05$ was considered significant.

\section{Results}

The mean birth age of the study group was $27.3 \pm 1.9$ gestational week (gw) and the control group was $28.9 \pm 2.0 \mathrm{gw}$. The mean birth age was significantly different between the groups $(P=0.036)$, but birth weight was similar in both groups. There was also a significant difference in gender distribution between the two groups $(P=0.021)$. Table 1 presents the characteristics of the

Table 1 Characteristics of the study and control groups

\begin{tabular}{lccr}
\hline Characteristic & Control (n:15) & ROP (n:13) & P-value \\
\hline Sex (male/female) & $5 / 10$ & $10 / 3$ & $\mathbf{0 . 0 2 1}$ \\
Age (gestational week) & $28.9 \pm 2.0$ & $27.3 \pm 1.9$ & $\mathbf{0 . 0 3 6}$ \\
Birth weight (gram) & $1153.2 \pm 367.9$ & $972.7 \pm 270.0$ & 0.156
\end{tabular}

Abbreviation: ROP, premature retinopathy. Bold and italic values show significant $P$-values. subjects. Plasma samples from 13 patients with ROP and 15 control subjects were examined for the expression of the 46 miRNAs using high-throughput qRT-PCR (Table 2). While the expression of miR-23a and miR-200b$3 p$ was significantly upregulated in patients with ROP when compared with the control group $(P=0.009$, $P=0.03$, respectively), the expression of $\mathrm{miR}-27 \mathrm{~b}-3 \mathrm{p}$ and

Table 2 Expression of plasma miRNAs in patients with ROP as compared to control group

\begin{tabular}{|c|c|c|c|c|}
\hline miRNA & $\begin{array}{c}R O P \Delta C t \\
(\text { mean } \pm S D)\end{array}$ & $\begin{array}{l}\text { Control } \Delta C t \\
(\text { mean } \pm S D)\end{array}$ & $F R$ & P-value \\
\hline let-7b-5p & $0.5 \pm 3.41$ & $0.01 \pm 5.37$ & -1.41 & 0.17 \\
\hline miR-23a-3p & $-2.65 \pm 1.16$ & $0.05 \pm 5.55$ & 6.5 & 0.009 \\
\hline miR-96-5p & $9.08 \pm 4.05$ & $7.82 \pm 5.71$ & -2.39 & 0.43 \\
\hline miR-92a-3p & $-3.48 \pm 1.6$ & $-2.59 \pm 5.7$ & 1.85 & 0.13 \\
\hline miR-199a-5p & $4.38 \pm 4.13$ & $1.98 \pm 6.03$ & -5.3 & 0.29 \\
\hline miR-296-5p & $5.77 \pm 7.05$ & $7.84 \pm 5.9$ & 4.2 & 0.2 \\
\hline let-7f-5p & $5.6 \pm 5.28$ & $4.62 \pm 6.3$ & -1.97 & 0.82 \\
\hline miR-26a-5p & $5.4 \pm 5.76$ & $5.5 \pm 5.14$ & 1.08 & 0.34 \\
\hline miR-99a-5p & $5.62 \pm 4.51$ & $3.36 \pm 4.32$ & -4.78 & 0.36 \\
\hline miR-146a-5p & $2.63 \pm 3.1$ & $2.4 \pm 3.56$ & -1.18 & 0.35 \\
\hline miR-200a-3p & $7.74 \pm 6.07$ & $9.39 \pm 3.86$ & 3.13 & 0.29 \\
\hline miR-328-3p & $2.33 \pm 5.92$ & $3.38 \pm 5.33$ & 2.08 & 0.27 \\
\hline miR-15a-5p & $-0.84 \pm 3.55$ & $-0.02 \pm 5.71$ & 1.76 & 0.67 \\
\hline miR-27b-3p & $2.46 \pm 3.61$ & $1.51 \pm 4.91$ & -1.92 & 0.011 \\
\hline miR-106a-5p & $0.3 \pm 4.31$ & $-1.31 \pm 6$ & -3.06 & 0.28 \\
\hline miR-150-5p & $2 \pm 3.47$ & $2.37 \pm 4.89$ & 1.29 & 0.39 \\
\hline miR-200b-3p & $6.21 \pm 3.37$ & $8.19 \pm 3.98$ & 3.94 & 0.036 \\
\hline miR-378a-5p & $6.34 \pm 8.31$ & $5.96 \pm 8.26$ & -1.31 & 0.29 \\
\hline miR-15b-5p & $1.26 \pm 4.05$ & $1.19 \pm 5.57$ & -1.04 & 0.29 \\
\hline miR-29a-3p & $3.69 \pm 4.25$ & $2.95 \pm 4.81$ & -1.67 & 0.47 \\
\hline miR-125b-5p & $1.8 \pm 5.14$ & $-0.41 \pm 4.03$ & -4.62 & 0.35 \\
\hline miR-155-5p & $8.77 \pm 3.05$ & $7.3 \pm 4.86$ & -2.76 & 0.1 \\
\hline miR-210-3p & $1.77 \pm 4$ & $2 \pm 5.16$ & 1.17 & 0.82 \\
\hline miR-410-3p & $1.46 \pm 2.35$ & $3.19 \pm 4.52$ & 3.32 & 0.1 \\
\hline miR-16-5p & $-6.87 \pm 2.42$ & $-6.23 \pm 4.02$ & 1.55 & 0.32 \\
\hline miR-30a-5p & $4.76 \pm 6.4$ & $4.4 \pm 5.1$ & -1.28 & 0.2 \\
\hline miR-126-3p & $-2.4 \pm 2.99$ & $-1.61 \pm 5.59$ & 1.72 & 0.26 \\
\hline miR-181a-5p & $1.65 \pm 3.3$ & $2.5 \pm 4.67$ & 1.8 & 0.32 \\
\hline miR-214-3p & $5.4 \pm 4.11$ & $2.52 \pm 4.74$ & -7.4 & 0.02 \\
\hline miR-424-5p & $0.6 \pm 4.76$ & $-0.26 \pm 5.76$ & -1.82 & 0.3 \\
\hline miR-17-5p & $5.23 \pm 7.25$ & $7.05 \pm 3.86$ & 3.52 & 0.12 \\
\hline miR-30e-5p & $1.9 \pm 6.07$ & $0.92 \pm 6.43$ & -1.98 & 0.29 \\
\hline miR-130a-3p & $2.19 \pm 4.29$ & $1.67 \pm 5$ & -1.44 & 0.43 \\
\hline miR-182-5p & $9.16 \pm 2.64$ & $9.56 \pm 3.64$ & 1.31 & 0.72 \\
\hline miR-218-5p & $8.55 \pm 2.41$ & $8.87 \pm 5.55$ & 1.25 & 0.29 \\
\hline miR-451a & $-6.46 \pm 2.08$ & $-4.73 \pm 7.32$ & 3.32 & 0.23 \\
\hline miR-20a-5p & $-1.78 \pm 4.45$ & $-1.37 \pm 6.19$ & 1.33 & 0.36 \\
\hline miR-31-5p & $6.91 \pm 3.31$ & $8.27 \pm 4.03$ & 2.57 & 0.54 \\
\hline miR-132-3p & $2.38 \pm 2.65$ & $4.79 \pm 4.36$ & 5.29 & 0.13 \\
\hline miR-183-5p & $-2.93 \pm 9.49$ & $-1.77 \pm 8.33$ & 2.23 & 0.3 \\
\hline miR-221-3p & $-0.07 \pm 3.48$ & $0.39 \pm 6.21$ & 1.37 & 0.2 \\
\hline miR-20b-5p & $6.09 \pm 7.03$ & $6.91 \pm 7.97$ & 1.77 & 0.4 \\
\hline miR-93-5p & $-1.2 \pm 3.63$ & $-1.22 \pm 4.77$ & -1.02 & 0.68 \\
\hline miR-140-5p & $7.22 \pm 4.04$ & $7.66 \pm 5.53$ & 1.36 & 0.29 \\
\hline miR-184 & $9.9 \pm 1.82$ & $11.13 \pm 2.5$ & 2.35 & 0.19 \\
\hline miR-222-3p & $2.16 \pm 6.02$ & $3.23 \pm 5.05$ & 2.1 & 0.26 \\
\hline
\end{tabular}

Abbreviations: FR, fold regulation; $\Delta \mathrm{Ct}$, delta threshold cycle. Bold and italic values show significant $P$-values. 


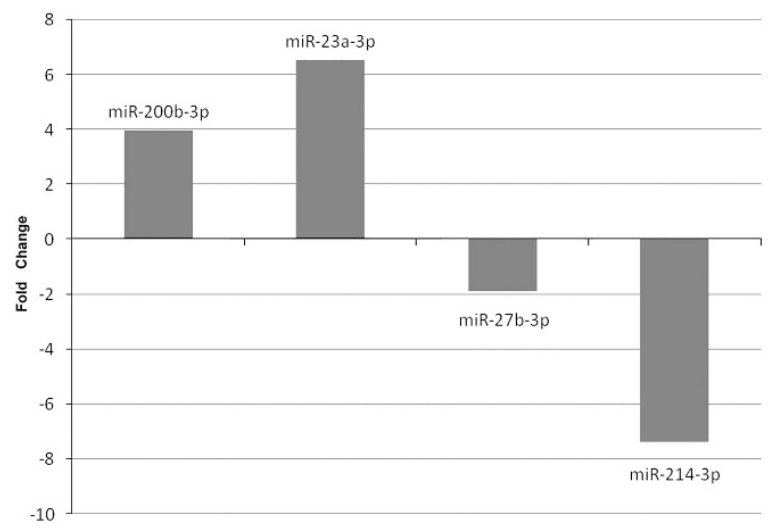

Figure 1 Significantly downregulated and upregulated miRNAs in patients with ROP compared with the control group.

Table 3 The genes on the pathway of angiogenesis targeted by miR-23a, miR-200b-3p, miR-27b-3p and miR-214-3p, expressed significantly using the Targetscan software program

\begin{tabular}{lll}
\hline & \multicolumn{2}{c}{ Target genes of miRNAs } \\
\cline { 2 - 3 } & miRNAs & Target Gene \\
\hline Upregulated miRNAs & miR-23a & Ism1 \\
& miR-200b-3p & VEGF-A \\
Downregulated miRNAs & miR-27b-3p & VEGF-B, VEGF-C \\
& miR-214-3p & HIF1AN, ANG \\
\hline
\end{tabular}

Abbreviations: Ang, angiogenin; HIF1AN, hypoxia inducible factor 1 alpha subunit; ISM1, isthmin1; VEGF, vascular endothelial growth factor. (www.targetscan.org; www.mirdb.org).

miR-214-3p was significantly downregulated in patients $(P=0.01, P=0.02$, respectively; Figure 1 ; Table 3$)$.

\section{Discussion}

In the literature the relationship between miRNA and ROP is based on experimental studies. In the present study we aimed to analyze the relationship between ROP and miRNA in patients and this aspect of the study has the distinction of being the first clinical study in the literature. If this relationship can be clearly demonstrated, early diagnosis and screening tests can be developed, and new treatment alternatives can be demonstrated in individuals genetically predisposed. Recent years, treatment approaches focusing on miRNAs are being studied particularly for diseases involving neoplastic and angiogenic process. ${ }^{10}$ Similarly miRNAs are thought to be potential therapeutic targets in ROP treatment.

Kutty et al showed that reactive oxygen species increased the expression of miR-23a from ARPE-19 cells. ${ }^{11}$ In addition, Lin et al suggest that miR-23a can be protective for ARPE-19 cells under oxidative stress. ${ }^{12}$ In the present study, we found that expression of miR-23a was significantly upregulated in patients with ROP. miR-23a expression can be induced by ischemic retina in ROP patients and can be protective for retina pigment epithelium.

It is known that there is a relationship between VEGF and ROP. In recent years, researchers have begun to analyze the relationship between VEGF and miRNA. They particularly began to bring up the miRNAs targeting mRNA which has effect on VEGF expression and the miRNAs targeting VEGF in receptor and signaling pathway. Zhao et al analyzed a group of miRNAs in newborn mouse with oxygen induced retinopathy and they related those miRNAs with VEGF. ${ }^{13}$ They identified both increasing and decreasing in expression levels of totally 66 miRNAs during oxygen induced retinal neovascularization. Comparing to present study, we similarly identified decrease in miR-378 and miR-29a expression and increase in miR-181a, miR-182, miR-183 and miR-200b expressions. But, we identified a significant increase only in miR-200b expressions in the patient group. Expression of miR-200b may be related VEGF secretion in ROP. However, Chan et al about found that miR-200b triggers angiogenesis targeting Ets-1 in endothelial cells. ${ }^{14}$ Similarly, miR-200b may be effective in ROP patients with this way, but it will be appropriate to reveal the active pathway with new studies. In another study, van Mil et al identified an increase in retinal vascularization and VEGF levels as miR-214 expression decreases. ${ }^{15}$ They particularly stated that miRNA-214 targets the mRNA of VEGF in gene level. But, Shen et al demonstrated an increase in expression of miR-214 in the ischemic floor of mouse retinas. ${ }^{6}$ In addition, it is known that $\mathrm{miR}-27 \mathrm{~b}$ can suppress angiogenesis via inhibition of VEGF-C in cancer tissue. ${ }^{16,17}$ In the present study, we detected a significant decrease in miR-214 and miR-27b expression in the patient group compared with the control group. VEGF level can be related with miR-214 and miR-27b expression in ROP patients.

Tea et al observed expression of miR-16 to be decreased in oxygen induced retinopathy with the increase of oxygen levels in retina. ${ }^{7}$ But in the present study, we found that miR-16 expression was similar in both groups. Chen et al detected increase in miR-410 expression and decrease in VEGF concentration during neovascularization in newborn mouse retina where ischemia was induced. ${ }^{18}$ They also anticipated that the increase in expression of miR-410 decreases VEGF levels. We did not observe significant change of miR-410 expression in ROP patients compared to control group. In addition, Nunes et al identified that the expression of miR-17, miR-20b, miR-93 and miR-106 decrease during the early phase of neovascularization in premature mouse which is formed in hypoxic ground, therefore they identified increase in VEGF levels. ${ }^{19}$ They also observed increase in miR-210 and miR-296 expression in the same 
study. In this study, these miRNAs were not significantly change in ROP patients compared to control group.

The genes on the pathway of angiogenesis targeted by miR-23a, miR-200b-3p, miR-27b-3p and miR-214-3p, expressed significantly using the Targetscan software program, were screened and summarized in Table 3. The balance between these miRNA expressions may have an active role in ROP pathophysiology. The increase in expression of miR-23a suppress the angiogenesis inhibitor Isthmin 1 and the decreasing expression of miR-27b-3p and $\mathrm{miR}-214-3 \mathrm{p}$ cause the increase of angiogenic factors. This may contribute to the formation of new vessels in ROP. However, the increase in expression of miR-200b-3p may suppress angiogenesis by reducing VEGF-A expression and may be associated with other miRNAs. However, it is appropriate to evaluate these possible pathways with new studies.

The difference between the two groups in terms of age of birth and gender distribution can be considered as a disadvantage of the study. Although the relationship between birth age and miRNA expression in premature infants has not been evaluated so far, birth age may be effective on miRNA expressions. It is known that in adult humans, the miRNA levels excreted in tissues change with age progression. However, it is not known whether there is a relationship between gender and miRNA expressions.

The relationship between miRNAs and angiogenesis of ROP and VEGF has not significantly revealed so far and the number of studies that focused on this relationship is limited. The best way to analyze this in a more detailed way is to conduct new studies on ROP patients. Moreover, it must be taken into consideration to develop a new type of scanning test focusing on miRNA subtypes that are significantly expressed in ROP patients. Based on this study, it will be reasonable to test the reliability of the results, with the studies involving more patient and control groups.

In conclusion we analyzed and compared the levels of miRNAs in ROP patients and control group, which are significantly expressed in angiogenesis and retinal neovascularization confirmed in literature. We suggest that these miRNAs may be potential candidates for novel biomarkers in ROP. Our results may be possible to develop new diagnostic and therapeutic approaches by means of multi-centered studies focusing on identifying miRNA levels.

\section{Summary}

What was known before

- Role of the VEGF in pathogenesis of ROP.

\section{What this study adds}

- Plasma miRNA levels may alter in ROP and, some miRNAs might have an effect in the physiopathology of this disease.

\section{Conflict of interest}

The authors declare no conflict of interest.

\section{Acknowledgements}

This study was supported by Mersin University Scientific Research Fund (Grant no: BAP-TF CTB (TM) 2014-1 TU).

\section{References}

1 Gilbert C, Rahi J, Quinn G. Visual impairment and blindness in children. In: Johnson GJ, Minassian DC, Weale RA, West SK (eds). Epidemiology of Eye Disease. 2nd ed. Edward Arnold Ltd.: London, 2003, pp 260-286.

2 Chen J, Smith L. Retinopathy of prematurity. Angiogenesis 2007; 10: 133-140.

3 Niranjan HS, Benakappa N, Reddy KB, Nanda S, Kamath MV. Retinopathy of prematurity promising newer modalities of treatment. Indian Pediatr 2012; 49: 139-143.

4 Agrawal S, Chaqour B. MicroRNA signature and function in retinal neovascularization. World J Biol Chem 2014; 5: 1-11.

5 Zhao R, Qian L, Jiang L. miRNA-dependent cross-talk between VEGF and Ang-2 in hypoxia-induced microvascular dysfunction. Biochem Biophys Res Commun 2014; 452: 428-435.

6 Shen J, Yang X, Xie B, Chen Y, Swaim M, Hackett SF et al. MicroRNAs regulate ocular neovascularization. Mol Ther 2008; 16: 1208-1216.

7 Tea M, Michael MZ, Brereton HM, Williams KA. Stability of small non-coding RNA reference gene expression in the rat retina during exposure to cyclic hyperoxia. Mol Vis 2013; 19: 501-518.

8 Mestdagh P, Van Vlierberghe P, De Weer A, Muth D, Westermann F, Speleman $\mathrm{F}$ et al. A novel and universal method for microRNA RT-qPCR data normalization. Genome Biol 2009; 10: R64.

9 Pfaffl MW. A new mathematical model for relative quantification in real-time RT-PCR. Nucleic Acids Res 2001; 29: e45.

10 Small EM, Olson EN. Pervasive roles of microRNAs in cardiovascular biology. Nature 2011; 469: 336-342.

11 Kutty RK, Samuel W, Jaworski C, Duncan T, Nagineni CN, Raghavachari $\mathrm{N}$ et al. MicroRNA expression in human retinal pigment epithelial (ARPE-19) cells: increased expression of microRNA-9 by N-(4-hydroxyphenyl) retinamide. Mol Vis 2010; 16: 1475-1486.

12 Lin H, Qian J, Castillo AC, Long B, Keyes KT, Chen G et al. Effect of miR-23 on oxidant-induced injury in human retinal pigment epithelial cells. Invest Ophthalmol Vis Sci 2011; 52: 6308-6314.

13 Zhao R, Qian L, Jiang L. Identification of retinopathy of prematurity related miRNAs in hyperoxia induced neonatal rats by deep sequencing. Int J Mol Sci 2015; 16: 840-856.

14 Chan YC, Khanna S, Roy S, Sen CK. miR-200b targets Ets-1 and is down-regulated by hypoxia to induce angiogenic response of endothelial cells. J Biol Chem 2011; 286: 2047-2056.

15 Van Mil A, Grundmann S, Goumans MJ, Lei Z, Oerlemans MI, Jaksani $S$ et al. MicroRNA-214 inhibits angiogenesis by targeting Quaking and reducing angiogenic growth factor release. Cardiovasc Res 2012; 93: 655-665. 
16 Ye J, Wu X, Wu D, Wu P, Ni C, Zhang Z et al. miRNA-27b targets vascular endothelial growth factor $\mathrm{C}$ to inhibit tumor progression and angiogenesis in colorectal cancer. PLoS One 2013; 8: e60687.

17 Liu HT, Xing AY, Chen X, Ma RR, Wang YW, Shi DB et al. MicroRNA-27b, microRNA-101 and microRNA-128 inhibit angiogenesis by down-regulating vascular endothelial growth factor $\mathrm{C}$ expression in gastric cancers. Oncotarget 2015; 6: 37458-37470.
18 Chen N, Wang J, Hu Y, Cui B, Li W, Wu G et al. MicroRNA-410 reduces the expression of vascular endothelial growth factor and inhibits oxygen-induced retinal neovascularization. PLoS One 2014; 9: e95665.

19 Nunes DN, Dias-Neto E, Cardo-Vila M, Edwards JK, Dobroff AS, Giordano RJ et al. Synchronous downmodulation of miR-17 family members is an early causative event in the retinal angiogenic switch. Proc Natl Acad Sci USA 2015; 112: 3770-3775. 